\title{
IV.
}

\section{Bericht über die Holländische ophthalmologische Litteratur des Jahres 1899 (2. Semester)}

\author{
von \\ Dr. (i. J. SCHOt'T'E und Prof. Dr. W. KOs'Tsik Gzn. \\ in Amsierdam. \\ in I.eiden
}

B. R. = Belang en liecht.

G. C. = Geneeskundige Courant.

II. B. = Hygienische Bladen.

K. A. W. = Verslagen der Koninklijke Academie van Wetenschappen.

M. G. T. = Militair Geneeskundıg Tijdschrift

M. W. = Medisch Weebblad.

N. O. B. = Nederlindsche oogheelkuudige bijdragen.

N. T. G. = Nederlandsch Tijdschrift roor Geneeskunde.

P. N. B. = P'sychiatrische en Neurologische bladen.

T. N. J. = Geneeskundig Tijdschrift voor Ned-Indii.

Die mit "rersehenen Nummern werden weiter unten referiert.

1. Borg, A. A. Hijmans van den. Coloboma iridis et chorioideae met vruegtijdig sluiten der fontenellen. (Iris- "nd Chorioideal-Colobon nit vorzeitigen Schluse der liontinellen.) X. T. G. II. S. is7..

2". Bemmelen, J. F. van. liesultaten van een vergelijkend onderzoek der verhemelte - orbitial - "n slaitpstreek ran den schedel der Monotremen. (Wrgebnisse einer vergleichenden Untersuchung der GaumenAlugenhählen- und Schläfengegend des Monotremenscbädels.) K. ג. W. S. $1 \%$.

3*. Blok, 1). .l. Over neutrale murias cocaine en sulf. atropine. (Neutrale Murias (Cocaini unil Sulf. Atropini.) N. (). B. Lief 8. S. 82.

4. Blok, D. I. Hypertrophia plicae semilunaris. N. O. B. Lief 8. S. 85.

5. Bouvin. M. Inrichting voor Uoglijders te's Gravenhage. Verslag over 1898. (Augenklinik im Halag, Bericht über 1898.) N. O. B. Lief 8. S. 111. (1146 l'at., 28 Kataract-()perationen.)

$6^{*}$. Brugh, J. I'. vall der, Twee stellingen nit de theorie van lieddingius.

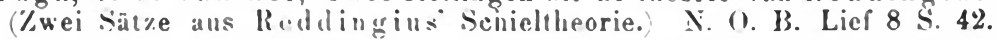

7. Brugh. d. P. van der. Een lens, wart door hoedkleurstof. Kine durch Blutfarlusiofie geschwiryte Linse.) N. () B. I.iof s. S. te.

8*. Gorkom. W. I. van, Over purpura variolosa. T. N. I. XXXld lief 4. S. 458 .

9. De II a as, .J. H. Vereeniging tot het verleenen van hulp aan minvermogende ooglijders rour Zuill-îlolland. Yerslibg ner 1898. (Angenklinik in liotterdam. Pericht über 1898. N. O. B. Lief 8 S. 110. (50)4 Pat. (33 Kataract-(1perationen.)

10*. Hoffmann. C. K., Bijdratge tut de kennis der ontwikkelingsgeschiedenis van den sympathicus. (Beitrige zur entwicklungsgeschichtlichen Kenntriss des Srmpathieus.) P. N. B. S. 3:2.

11. Koster, W. G\%n., De verhouding Van den Musculus tarsalis superior Mülleri hij ptosis congenita. (I)ie Verhältnisse des Musc. Tarsalis superior Ilülleri hei congenitaler Ptosis.) N. T. G. II 417. (Wird spater in dieser \%eilschrift mitgetheilt werden.)

12. Hoster, W. (izn.. Eene methode ter operatic van ptosis. (Eine noue P'tosis-0peration.) N. T. G. II 8.33. (Vol. '/eitschr. f. Aughk. 1889, S. 543.) 
13. Lans, 1. J.e (Ner opticus-facialis reflex met daarbij roorkomende refractaire phase. (Uebur Opticus-liacialis-Reflex und cine refractäre ('hase bei demselhen.) N. (). B. l,ief 8. S. 86 .

14\%. Lan s. I.. J., Pupilwijolte. (Pupillenweite.) N. T. G. IJ S. 114i.

15. $\mathrm{M}$ ulder. M. E.. In richting tut behandeling en verpleging van behoeftige en minvermogende ouglijders te (irnningen. Verslag over 189s. (Augenklinik in Groningen, Bericht ïber 1898.) X. O. B. J.ief 8 S. 110. (1762 Pat., 3() liataractoperationen.)

16:k 11 ulder, M. F.. Blepharitis ciliaris en acarus of demodex folliculorum. N. I'. G. I1. S. SO3.

17. Muntendam. P'. lien geval van chinine-blintheid. (Fin Fall ron ChininBlindlieit. sehr genau lieschrieben und den Typus gan\% entsprechend, namentlich was das Gesichtsfeld anbelangt) N. O. B. lief 8. S. 73.

18. Muntendam. P. und Vries, W. M. de, IX internationat oogheclkundig Congres. Verslag der sectievergaderingen. (Bericht über die sil\%ungen dor Sectionen des Utrechter Coungresses.) N. T. G. Il. S.674.

19. Muskens, 1. J. J.. Twee gezwellen vau medulla en pons. (\%wei Tumoren von Medulla und l'ons; ein mit Autopsie, tin mit günstigem Verlauf: ein librusarcom, ein ungewisser.) P. N. B. S. 615.

20\%. Nicolai, C., Du \% g. schaduwproef. (Skiaskopie.) X. (). B. I.ief \& S. 30.

21. Pel, P. K.. 'Tumor cerebelli. N. 'T' (i. II. S. ]2-27.

22. Pinkhof. H.. Een model oogengasthuis. (Das neue linyal Lundon Ophthalmic Hospitil.) N. J. G. II. S. $\$ 11$.

23*. Plaats, J. I). Pan der. Over het lichten van het oog. (Das Augenleuchten.) Versamml. des Provinciaal Uirechtsch Genootschap r. Kunsten en Wretenschappen, 5 Juni.

247. Plaats, J. D. van der. liene natuurkundige verklaring ran de schaduwprot. (Kin phrsische lirklärung (ier Schattenprobe.) N. O. B. Lief S. S. ¿).

25. Roddingius, R. A.. Alg. Haag sche Poliklinick. Verslag over 1898 (Klinik in Hatag.) N. O. B. Linf. \& S. 111. (57!) Pat.)

$26^{*}$. Reddingius, K. $\Lambda$., Antwoord op de vourdracht van Dr. Van der Brugh. (Erwiderung an Herrn Di. Iau der Brugh.) N. (\%. B. Lief 8 S. 5 \%.

27. Reddingius, R. A., Esuphoria als oor\%ak vin schonlinyopie. (lisophorie eine Ursache der Schul-Myopie.) (i. (. No. 8.

28. Schoute, G. J., Een geval van curnea-verwunding met perforatie der iris zonder beschadiging van de lens. (Ein Fall ron Cornealruptur mit Irisperforation ohne Verlet\%ung (ler Linse.) N. T. G. 11. S. 298. Vergl: Zeitschrift für Augenheilkunde | S, 374.

29. Schoute, G. J., Sclerailruptuur. (Skleralruptur, suture en bourse (Kuhnt) Heilung.) N. O. 13. Licf 8. S. 73 .

$30^{*}$. Sissingh, R.. De algemeene eigenschappen der optische afbeelding door centrale stralen in een reeks rar. gecentreerle bolvormige oppervlakken. (Die allgemeinen Kigenschaften der optischen l)arstellnng durch centrale Strahlen in einer Reihe centrirter kugelfürmierer Obertlächen.) K. 1. IV. S. 258.

31*. Snellen, H. Jr. Discussion über Vin der Plata Theoric der Schattenprobe. 犬. O. B. Lief 8 . S. 28 .

32\%. Snellen, H. Sr., Blepharitis e demodice. N. O. B. Lief S S. $\mathbf{8 5 .}$

33. Snellen. H. Sr. Nederlandsch Gasthuis vour ooglijders te Eitrecht. Verslag over 1598. (Niederländisches ophthalmologisches Krankenhaus in Utrecht. Bericht üluer 1898.) N. 'T. G. II. S. 642. (5796 J'at.. 99 liataract-(Operationen.)

34. Snellen, H. Sr., De voltoviing van het Nederlandsch gasthuis vor ooglijders. (Die Vollendung des Niederlärdischen ophtalmologischen Krankenhauses.) Utrecht 1899.

35. Snijders, 1. I. (., Iets over het rooken. (Das liauchen.) H. B. S. 305. 36. Snijders. A. I. (., De verlichting van school en huis. (Die Beleuchtung in der schule unil z.u Hause.) B. R. J)e. 
$37^{*}$. Stort. A. H. van Genderen. Teleneuronen in het netrlies van leuciscus rutilus. (Teleneuronen in der Net\%haut von I.cuciscus rutilus.) N. T. G. II. S. 270 .

$38^{*}$. Straub. Il., Behandeling der gordelvlekten op de cornea. (Behandlung der Bandkeratitis.) N. T. G. II \& j93.

39. Straub. M., Blindheid bij chinine-vergiftiging. (Blindheit durch ChininIntoxication.) N. T. G. II. S. T4t.

40. Straub, M.. Opneming van tluorescine (lnor curneac zonder epithecldefecten. (Fluorescin-Resorption ohne Epithelverlust der Hornhaut.) N. T. G. Il. S. 1227 .

$41^{*}$. Straub, M.. De indicaties voor onderbuidsche inspuitingen met sublimaat. (Die Indikationen der subcutanen Sublimatiujektionen.) N. T. G. II S. 1247 .

42. Terburgh, I T. Statistische beschouwingen over de in de jaren 1996 en 1897 voorgekomen ziektegevallen onder de dwangarbeiders te Sawahlocnto. (Statistisches über die Krankheiten der Zwangsarbeiter in einsor Mine von Niederl. Ost-Indien.) 'T. N. J. XXXIX Lief \& S. 37\%. (\%iemlich viel Bindehaut- und Mornhauterkrankungen, theils absichtlich, theils durch Sprengen des Bergwerks mittelst Pulver.)

43. Visser. B. P., Geschiedkuudig overzicht van de bepalingen omtrent gezichtsscherpte en refractie in onze keuringsreglementen. (Geschichtliches hetrettend die lerorduungen üher Sehschärfe und Refraction in unseren gesetzolichen Prüfungsvorschriften.) M. (i. 'I. Lief 3. S. 1.18 .

44. Iries, W. M. de, De musculus dilatator pupillac, met de nieuwste kleur. methoden aangetrond. (Musculus I)ilatator P'upillac. dargezeigt nit den neuesten liärbun يrsmethoden.) N. (). B. Lief 8. S. $6 \dot{3}$.

4i). Westh off, C. H. A., l'estrele für Herrn l'rof. W. M. Gunning. N. O. B. Lief. 8. S. 37 .

46. Westhoff, C. H. A.. Distichiasis congenita hereditaria. N. (). B. licf 8. S. $6 \dot{\text {. }}$.

47. Westh off, C. H. A., Een guval vin aungeboren dubbel\%ijdige lensluxatie. (Congenitale doppelseitige liusenluxation) N. 0. B. Lief 8. S. 66 .

48. IVesth off, C. H. A.. Herinneringen uit de laatste vijftig jaar der oogheelkunde. (Erinnerungen aus den letzten fünf\%ig Jahren der Augenheilkunde.) H. IV. S. 20t.

49. Westh off, C. H. A., liostelooze Ansterdamsche polikliniek. (Poliklinik in Amsterdam.) Bericht ïber 1898. (1)39 l'at., 18 Kataratet-Operationen.)

50. Westhoff, C. H. A., Protargol in de oogheelkunde. (Protargol in der Augenheilkunde.) M. IV. Dez.

j1. Wiersma, li.. lien tumor in do pons. (Pons-tumor.) P. N. B. S. 603. ('luberkel.)

$522^{2}$. $Z$ wa a rdemaker. H., (Over een subcorlicalon retlex en een daarbij voorkomende rofractaire phası. (Ein subcorticaler lieflex undeine refraktäre Phase bei denselben.) N. T. G. II. S'. 844 .

Nach Untersuchungeu lan Bemmelen's ( 2 ) bildet ein grösserer, vorderer, senkrechter Flügel des Gaumenbeins einen Theil des Augenhöhlenrandes; der Vorderrand eines kleineren, hinteren, senkrechten Flügels begrenıt die Aussenseite der zu einer Oefinung verschmol\%enen Foramina rotundum, opticum und sphencorbitale (Echidna). Ornithorrhynchus hat ein separates foramen rotundum. Monotremata haben riesenartige Orbito-sphenoidea (d. h. Alae minores), welche mit dem Keilbeine verwachsen sived.

Il offmann (10) entuimmt seinen Untersuchungen, hauptsächlich an Acanthias vulgaris, dem Dornhai, folgende Schlüsse:

Sympathische Gauglieu entstehen wach 'issammenkunft der ventralen Nervenwur\%el mit der dorsalen, welche let\%tere, in Rumpfgebiete, ver- 
läuft zwischen Lrwirbel und Rückenmarkskanal. Am Kopfe fehlt die dorsale Wurzel beim 10., 9. und 8 . L'wirbel, die ventrale beim $7 ., 6$. , 5. und 4. Der 3. Urwirbel hat ventral den $\mathrm{N}$. VI und dorsal Zweige der N. N. VII-VIII; dieser let»tere aber rerläuft, wie alle dorsalen Kopfwurzeln, ausserhalb des Urwirbels, wodurch ein Zusammentritt mit der ventralen Wur\%el unmöglich wird.

l)er 2. Urwirbel hat keine ventrale $l$ ur\%el (der $\mathbb{N}$. $I V$ ist eine dorsale).

Der I. Lrwirbel hat rentral den $\mathbf{N}$. III und dorsal den $N$. ophthalınicus prof. (gehört zum N. V), der einzige dorsale Gehirnnerv, der rein sensibel bleibt (bis \%um Menschen): diese \%usammen bilden das rein sympathische Ganglion ciliare.

'Thatsächlich fehlen die motorischen Wurzeln nicht am kopfe, sondern sie vereinigen sich wahrscheinlich schon im Centralorgan mit der seusiblen Wurzel: desshalb sind die ïbrigen kopfganglien nicht nur cerebro-spinale, sondern \%ugleich sympathische: was auch daraus hervorgeht, dass aus diesen Ganglien sympathische fasern zichen zur Gland Thymus, zum kopfdarm und zu den Kiemenarterien.

Höhere Thiere bekommen separate șmpathische Kopfganglien durch Sonderung von den gemischten.

Aus Van Genderen Stort's Arbeit (37) wollen wir die von ihm betonten lnterschiede seiner Befunde nit denjenigen Ed. P'ergens' (Action de la lumiere sur la rétine, 1897) hervorheben: Iccuciscus rutilus (die Plötze) besityt nur eine einfache Zapfenart, welche als kriftige Striinge bis durch die äussere granulirte jchicht. hindurch verfolgt werden kann. Die Stäbcheu sind kur\%.

Fine vergleichende Intersuchung eines Dunkel- und eines lichtAuges desselben Thieres ergah gleiche Dicke der Netzhaut in beideu Zuständen. In den Kernen der iiusseren granulirten Schicht wir keine Chromatin-Verminderung durch licht wahrnehubar. - Die schönsten Präparate bekommt Van Genderen Stort durch llirtung in 3,5 pCt. Salpetersäure-Lösung (in Holland höchstens 24 Stunden, in wärmerem Klima kïrzer) und Nachhärtung in M iiller's Augenfliissigkeit.

Reddiugius (26) meint, dass Accommodations- und ConvergenzInuervationen in unlöslicher Verbindung mit einander stehen (das fiegentheil war Donder's Meinung. Lis muss demzufolge die Fixation in P. R. immer mit einer minimalen Convergen\%-Innervation stattinden: und war auch dann, wenn man Prismen ror die Augen stells, weil dadurch die Accommodations-Anstrengung nicht geändert wird: daraus folgt, dass die binoculäre lixation lann nur noch möglich ist durch Aenderungen der Divergenz-Inuervation. IDiese letztere liann also berechnet werden aus der liraft ler l'rismen. welche in dieser "Weise überwunden werden künnen. - Die Convergen\%-Motilitiit wird gemessen durch den linterschied der stïrksten abducirenden Prismen. welche binoculiire fixation zulassen, 1. im P. R.. 2. in P. P.: In beiden Fällen ist die 1)ivergen\%-Innerration minimal, d. h. die gleiche. und der Interschied also blos auf Rechunug der Convergenz-Innervationen zu stellen.

Die Convergenz-Motilitiit wird gefunder: dreimal grösser \%u sein als die Divergenz-Notilität. 
Van der Brugh (6) nennt diese Messungsmethode nicht zulässig: man soll die absolute liraft der I)ivergen\% messen indem man ron dem am meisten convergenten Stand des Bulbus ausgelit und die Kraft der Convergenz in umgekehrter Weise: beide müssen dann einander gleich sein.

Wr fragt weiter, wie man je convergiren könnte, wenn. wie Reddingius meint, Convergen\% und l)ivergenz immer gleicli stark inuervirt wiirden: sind doch bekanntlich die M. M. recti externi die stärbsten und haben die günstigste Insertion.

Und falls eine uaximale Convergenz von einer maximalen Divergenz-Innervation begleitet sein muss, kann man sich keine noch stärkere Divergenz-Innervation denken, welche eine nächstfolgencle Fixation in der Ferne besurgen künnte.

Reddingius denkt sich dies folgendermassen möglich: 1. lixation in der Nähe, 2 . aufhöhren aller Fixations-Innervation, 3. Divergirende Finstellungs-Innervation, 4. Fixation in der Ferne.

lan der Brugh nimmt an: $1^{0}$ ein schwaches Dirergenz-Centrum (dazu gehracht durch die Symptome ler Jivergen\%-Paralyse (Straub) Reddingius nennt dasselbe ein starkes (entrum) $2^{0}$ ein kriftiges Convergenz-C'entrum, 3. Als Ruhestind der Augen eine leichte Divergenz. Nach Reddingius nimmt die liraft der Convergenz-Motilitiit unit dem Alter ab wie die Accommodation.

Lans 14) hat seine lintersuchungen über die Pupillenweite fortgesetzt. (Vgl. Bericht ïber d. 1. Sem. 18:9.) Bei einer Beleuchtung ron $0-25$ Mleterker\%en (Kunstlicht) wurde die Pupille photographirt nach Adaptation und ror dem Anfange des Pupillenreflexes; hei einer Beleuchtung ron 25-1000 Meterker\%en (Tageslicht) wurde sie gemessen mit Exner's Jaryngometer: Die Pupille verkleinert sich bei zunehmender Beleuchtung stets langsamer von 7,26 bis $\ddot{3}, 2 j \mathrm{~mm}$. Zwar schliessen sich die Aufnahmen bei liunstlicht denen bei 'Tageslicht vicht genau an, aher das war wegen Beschrinkung des Gesiclatsfeldes bei der ersten Methode auch nicht \%u erwarten. I,ans vermuthet ein inniges liand zwischen der Pupillenweite und der Sehschärfe. Die betonte Uebereinstimmung der diesbezüglichen graphischen Vorstellungen fand aber Ref. wenig auffallend, besonders nicht wemn man entweder die Pupillenoberfläche mit der quadratisclien Selsschärfe, oder den Pupillendurchmesser mit ler linearen sehschärfe vergleicht: was doch genauer Lans liedanken entsprechen muss als die von ihm selbst gegebene Darstellung.

l.ans achtet das Weber'sche (ieset\% auch für den motorischen lidreflex gültig.

Das bei diesen ('ntersuchungen entdeckte zeitweise Ausbleiben des lidreflexes - wïhrend der refralitären Phase - wird folgendermassen (13) unter leitung \%waardemikers (52) untersucht: Zur llervorrufung des optischen lidreflexes wird als Reiz der electrische funken benut\%t: der Lidschlag unterbricht einen electrischen Strom zur Registrirung der Bewegung. - Zwei l.ichtreize 0.7 Sec. nach einander, sind von \%wei Reflexell (lidschlägen) gefolgt: zwei Reize 0,6 Sec. nach einander (auf jedem Auge einer) lüsen nur einen Reflex aus: nach dem ersten Reize faingt eine refraktaire Phase an, welche $0,4-0,6 \mathrm{Sec}$. an- 
dauert, bisweilen cine Sec. und länger. (Der zweite Rei\% findet das Auge schon wieder geöffnet.) Zwei sehr schnell auf einander folgende Reize lüsen einen Reflex aus, der später kommt als gewöhnlich.

Der meclianische Lidreflex wird untersucht durch Anblasen der Hornhaut mit einem erwiirmten I,uftstrome. Hierbei dauert die refraktäre Phase 0,3 Sec. höchstens. Ein optischer Reiz, von einem mechanischen gefolgt. gibt zwei Reflexe. - Diese Unterschiede führen zu dem Schlusse, dass die refraktäre Phase ihren Ursprung nicht hat in einer Station, die dem optisclien und dem mechanischen Reflexe gemeinsam ist (im verlängerten Mark): auch nicht im Facialis-Kern, weil man das Auge nach dem Reflexe geschlossen halten kann, aber wahrscheinlich in den vorderen Vierhügeln, wohin die Reize von beiden Augen zusammenkommen. Auch bei den Kinie- und anderen Reflexen vermuthen Lans und Zwaardemaker eine refraktïre Phase.

sissingh (30) hat die Methode Bossclia \%ur Bestimmung der optischen Coustanten (K. A. W. 1879 und Annales de l'école nolytechnique 1886) angeweudet für neue Untersuchungen.

Nach Blok (3) wirken schwach sauere Collyrien Cocain, Atropin, Pilocarpin) auf vielen Bindehäuten stark rei\%end; auch die ron Koster beschriebene Cocain-Conjunctivitis (vgl. Bericht 1. Semester 1899) möchte eine Säure-Conjunctivitis seiu. Man verschreibe das Atropin in basischer lösung. das Cocain in neutraler.

Straub (38) berichtet über die Abrasio Corneac bei Bandkeratitis in gesunden Augen (er emptiehlt dazu das Von (iräife'sche Messer); er theilt mit, dass die 'Trühungen in und unter der Menbr. Bowmanni durch unregelmässigen Astignatisnus den lirfolg zwar beeinträchtigen, dass aber der letztere nach einigen Jahren verschwindet (die Seh schärfe hob sicb in einem Falle von ${ }^{1 / 60}$ nach der Operation bis zu ${ }^{1 / 2}$ drei Jahre später). - Die Abrasio ist auch angezeigt wenn sich schmerzliche atonische Geschwüre in der Trübung vorfinden. - Derselbe (41) Autor vertheidigt die subcutanen Sublimat-Injectionen: Sie sind angezeigt, wenn das Quecksilber seine Wirkung schnell entfalten soll: heftige Ureitis, luetische Neuritis optica; und wenn es als Probe gereicht wird: man braucht da\%u kleine J)oses und ist schon in einer Woche über seive Wirbung unterrichtet Aus Rüchsicht auf ansteigende Schmer\%furcht werden die Einspritzungen nur eine Woche ausgefiihrt: tiiglich 1 hisweilen $2 \mathrm{mal} 10 \mathrm{mgr}$ Sublimat als $1 /$ iller und Stein's Chlornatrium-Sublimat-l,ïsungen, mit Sorge fiir die Zaihne Zahnstein. Mundwasser und Controlirung des Harns und der 'Temperatur.

Mulder 16) beschreibt den Bau und die Entwicklung der Acari (drei Metamorphosen) und ihr Vorkommeu beim Venschen: ausser bei Blepharitis ciliaris syuamosa fand er sie bei Blepharitis ciliaris furfuracea und selbst bei Blepharitis ulcerosa: auch in recidivirenden Horleolen. lir vermuthet sie bei mehr als 2 pCt. der gesunden Jider. Cilien mit tordirter Wurmel und stark pigmentirtem besenartigen Bulbus lailt er gegenüber Ruehlmanu - nicht für pathogncmonisch, weil er sie auch unter gesunden Kopthaaren und Augenbrauen vorfand. - Balsamum perurianum liess ihn immer im Stiche, auch bei der genauesten (dazu ruch ziemlich schmerzvollen) Behandlung; wochenlaug fortgesetzt uuil 
durch Epilation unterstützt. - Snellen st. (32) empfiehlt ausser PeruBalsam: Quecksilber-Vaselin.

Der von Van Gorkom (8) beschriebene fall von Purpura variolosa wurde eingeleitet durch Blutungen unter der Lid- und Bindehaut; später kam da\%u Chemosis und Protrusion der purpurrothen Augen. Exitus letalis.

Van der Plaats $(23,24)$ erklärt die Skiaskopie in folgender Weise: 1. Was geht auf der Netzhaut vor? - Die Lichtquelle beleuchtet einen 'Theil der Netzhaut: dieses "Lichtfeld" bewegt sich, wenn der Spiegel gedreht wirl. Der l'ntersucher überblickt ein "Gesichtsfeld" dessen (irösse durch die Pupille des lintersuchten und durch die Spiegelioffnung bestimmt wird. Dieses (iesichtsfeld hat einen diffusen Rand: am schärfsten begrenzt ist es, weun der spiegel im P. R. steht. Wenn der Spiegel gedreht wird, bewegt sich das fiesichtsleld nicht. 2. Was sehen wir in der Pupille? -- Wenn richtfeld und Gesichtsfeld einander decken, hommen von jedem Punkte der Netzhaut Strahlen \%urück durch die ganze Pupille: sie gehen anch alle durch die Spiegelöfinuvg: daber erscheint die ganze Pupille leuchtend.

Wird nur der diffuse Rand des Gesichtsfeldes darch das lichtfeld beleuchtet, dann kommen von diesen beleuchteten Punkten zwar auch Strahlen durch die ganze Pupille \%uriirk, alier nicht alle gehen durch die Spiegelöffnung: sodass ein Theil der Pupille dunkel erscheint. Je nachdem das Bild der spiegelöfinung ror oder hinter der Netzhaut liegt. ist diese oder jene Seite der P'upille beschattet.

Ist der diffuse Rand am kileinsten (Spiegel im P. R.), so geht der Schatten am schnellsten an der Pupille vorüher d. h. plütrlich verdunkelt die l'upille gänzlich. (Wäre das Auge ohue optische Fehler, so würde nicht dieser lehergang noch schueller vor sich gehen, aber gar kein schatten auftreten.

Die Theorie wird in allen Intertheilen in mathematischen formeln ausgedrückt. dahei auch die Forn von spiegelöfnung und Pupille, und der Astignatismus herïcksichtigt: für alle Fialle wird eine Methode angegeben, un das was auf der Net\%haut vorgeht. auf die Pupille des Untersuchteu z.u projertiren.

leber einen anomalen Schatten vgl. Bericht ibler $189 \mathrm{~s}$.

Die Richtung des Schattenrandes fïllt nur daun ousammen mit einem der Hanpluteridiane den sehriben Astigmatismus. wenn der Spiegel im P. R. steht.

Van der Plaats emptiehlt einen grossen Planspiegel mit kleirer Oefinung, eine lichtyuelle nit scharfen Rindern und Vintersuchung in $1 \mathrm{~m}$ Entfernung. - \%ur Iemonstration werden verschiedene Cameralïinrichtungen angegeben.

Snelleu jr. (31) zieht es vor, den Gang ler lichtstrahlen, welche von einen einzigen beleuchteten Punkte der Net\%haut durch die Pupille des Cintersuchten und des Untersuchers treten \%u construiren wie dies l.e Roy angegeben und nachher diese Construction auszubreiten für einen nicht-punktförnigen Theil. Das Resultat ist das nämliche, weil die Pupille des Untersuchers benutzt wird anstatt deren Bild auf der Net\%haut des Intersuchers. Nach Nicolai (20) scheitert Van der Plaats' 'Theorie an folgenden Versuch: Man skiaskopire eine Camera 
mit einem Planspiegel und einer breiten Flamme: der Spiegel wird aber nicht gedreht, sondern die Flanme. der Spiegel und das Auge gleichmässig hin und her geschoben: auf dem Okular sieht man die skiaskopische Frscheinung und auf der Milchplatie der Camera sieht man, dass das Lichtfeld fortwährend das ganze Gesichtsfeld deckt, und nicht, wie Van der Plaats' Theorie fordert, nur den diffusen Rand desselben.

Er erklïrt dic Skiaskopie durch Bewegung des Flammenbildes über das Bild der Pupille des Untersuchten, beide im Auge des Untersucher:

\title{
V.
}

\section{Bericht über die ungarische ophthalmologische Litteratur.}

\section{(II. Semester.)}

Erstattet rou

\author{
Privatdocent Dr. ENII r. GRÓSZ-Budapest.
}

1. Mittheilungen aus der dugenklinik der Kön. ung. Universität in Budapest. (Jirektor Prof. Wilhelm Scbulek.)

1. v. Grós\%, Emil, Die Atrophie des Sehnerven bei Tabes dorsalis. Mathematikai és termés\%cttudományi értesitö, XVII, 1899, ref. im Ergün\%ungsheft der \%eitschrift für Augenheilkunde. II.

2. Derselbe, Nothwendigkeit der Gründung ron Provinzial-Augen-Heilanstalten. (Ein Antrag von Firiedrich Gros\% an der Wanderversammlung der ungarischen Naturforscher und Aer\%te im Jahre 1846.) Orvosi Hetilap, S\%emés\%et $1899 . \quad$ No. 5.

3. v. Blaskovics, Fridolin, Die offene Wundbehandlung nach Augenoperationen. Ibidem. No. 6.

4. Leitner, Wilhelm, Augensymptome bei Acromegalie. Ibidem.

5. Binder, Emil, Astigmatismus und dessen Untersuchungsmethoden vom Gesichtspunkte der Militärärzte. Ibidem. No. 4.

6. Pólya, Eugen, Tyloma (Keratosis) conjunctivac. Ibidem. No. (i.

\section{Audere Mittheilungen.}

7. Imre, Joseph, Die Ursachen der Blindheit im ungarischen Niederlande. Ibidem. No. 6.

8. Feuer, Nathan iel, Die operative Behandlung des Trachoms. (iyógyzašat. 1899. No. 44.

9. Derselbe, Die der\%eitigen Distichiasis-Operationen in der Trachomabtheilung des städtischen Krankenhauses St. Stephan. Budapest székes fuviros Közkórház:inak évkönyve

10. Goldzieher, Wilhelm. Buiträge zur Lehre: der Neubildungen des Auges: a) Fibron des Oberlides, verbunden mit Riesenwuchs der Haut des Gesichtes (erschienen in extenso Centralblatt für Augenheilkunde 1895, 17t: b) Degeneratio fibromatosa interstitialis retinac. Ueber einen bisher noch nicht bekannten Augenspiegel-Bofund. (lirschienen in extenso: Centralblatt für Augenheilkunde 1899. Märr.): c) liin Fall von Granuloma corneae. Ibidem.

11. Derselbe, Leber den Schulbesuch der schwachsichtigen Kincler. Orvosi Hetilap. Gyernekứgúgýs\%at. 1899. No. 3.

12. Csapodi, Istran, Die Ëintheilung der Myopie. Orvosi Hetilap S\%emés\%et. 1899. No. jo. 